\title{
Silicon Micro/Nanomechanical Device Fabrication Based on Focused Ion Beam Surface Modification and KOH Etching
}

\author{
J. Brugger ${ }^{a}$, G. Beljakovic ${ }^{b}$, M. Despont ${ }^{a}$, N.F. de Rooij ${ }^{b}$ and P. Vettiger ${ }^{a}$ \\ a'IBM Research Division, Zurich Research Laboratory, Säumerstr. 4, 8803 Rüschlikon, Switzerland \\ ${ }^{b}$ Institute of Microtechnology, University of Neuchâtel, Jaquet-Droz 1, 2007 Neuchâtel, Switzerland
}

Selective $\mathrm{Ga}^{+}$ion implantation and milling by focused ion beam exposure and subsequent wet chemical etching is used to fabricate micro/nanomechanical elements in $\mathrm{Si}$. Freestanding elements with a $\approx 30 \mathrm{~nm}$ membrane thickness are made by controlled selective underetching between unexposed and exposed areas. Ultrahigh-frequency cantilever beams have been made with resonances in the tens of $\mathrm{MHz}$ range. Using a U-shaped beam cross section, mechanical stiffness could be increased 100-fold, which in turn increased the beam resonance frequency to several hundreds of $\mathrm{MHz}$. The direct-write patterning/milling technique was used to fabricate various arbitrary shapes with vertical sidewalls such as submicrometer-sized containers, cups, and other nanomechanical devices.

\section{INTRODUCTION}

Miniaturization of mechanical devices is important for scaling the device density and its mechanical properties. For example, a cantilever with high resonance frequency and a low spring constant can only by achieved by reducing its mass. Many prospective parallel sensing systems, such as integrated optoelectronic/photonic devices, high-resolution matrix mirror displays, mechanochemical sensor arrays, and high-resolution data storage systems, require a high density of mechanical elements on a very small area. To achieve this, it is necessary to reduce further the size of the devices that are feasible with today's micromachining techniques, which is a challenging endeavor from a fabrication point of view.

Recently, a technique using the combination of local surface implantation by $\mathrm{Ga}^{+}$ions using a focused ion beam (FIB) and wet chemical etching has attracted much interest [1-3]. It is well known that a high impurity concentration in $\mathrm{Si}$ renders the implanted region relatively immune to subsequent etching with certain chemicals, such as potassium hydroxide (KOH) $[4,5]$. The advantage of using a FIB is that the ions can be exposed locally by direct-write patterning. Despite the disadvantage of being a serial process, it allows the fabrication of arbitrarily shaped devices down to the nanometer range. Underetched structures have been fabricated by appropriately oriented patterns $[1,3]$. Their structural thickness is determined by the penetration depth of the ions, which amounts to typically $30 \mathrm{~nm}$ for $\mathrm{Ga}^{+}$ions at an acceleration voltage of $30 \mathrm{kV}$ [2]. Interestingly, when milling a hole into the Si by continued FIB exposure, the remaining sidewalls become equally doped due to back scattered ions and hence become also etch resistant. This makes it possible to fabricate arbitrary three-dimensional elements with vertical extensions, such as sidewalls. In this paper we exploit this fact to improve the mechanical stability of ultrathin beams by milling a $U$ shaped cross section with an increased moment of inertia.

\section{FABRICATION}

\subsection{Focused Ion Beam Patterning and Milling}

We use $(100)$ oriented $\mathrm{Si}$ wafers with a $10 \Omega-\mathrm{cm}$ $n$-type background doping. The FIB system [6] is operated at $30 \mathrm{kV}$ ion beam acceleration voltage with currents in the range of $12-150 \mathrm{pA}$ that are suitable for our purposes in terms of beam resolution, dose, and write time. An advantage of the FIB lies in the nature of the process itself because the direct-write patterning simultaneously creates the mask layer, i.e. no intermediate mask pattern transfer step is necessary. The minimum 
(a) shallow doping with Ga+ ions

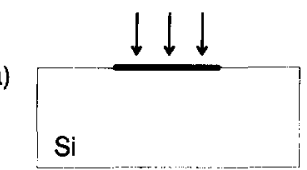

(b)

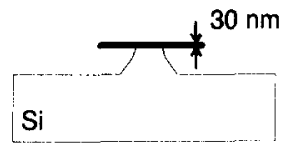

(c) rectangular cross-section

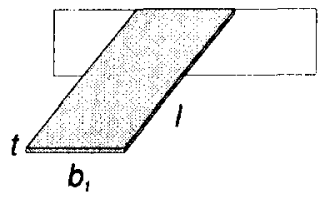

milling with $\mathrm{Ga}+$ ions and side-wall doping
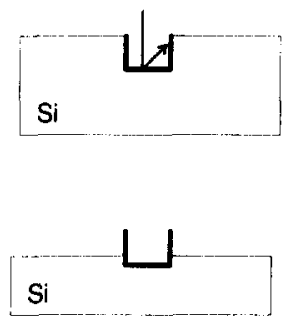

u-shape cross-section

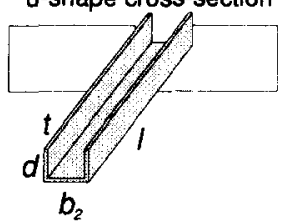

Figure 1. Fabrication of freestanding nanomechanical elements by shallow doping (left) or milling and sidewall doping (right) in various fabrication stages; (a) after FIB exposure, (b) during $\mathrm{KOH}$ etching and (c) when etching is completed.

dose required to render an area effectively insoluble in $\mathrm{KOH}$ is $10^{15}$ ions $/ \mathrm{cm}^{2}$ [1]. For example, a $5 \times 5 \mu \mathrm{m}^{2}$ area exposed to a current of $100 \mathrm{pA}$ requires only a few seconds to reach the level for the onset of the etch stop.

Material exposed to FIB is simultaneously removed, hence a continued exposure results in a pronounced material removal in this area (for $100 \mathrm{pA}$ we achieve a removal rate of the order of $0.25 \mu \mathrm{m} / \mathrm{min}$ ). Figure 1 shows schematically the basic patterning procedures for both the shallow doping and the milling approach.

\subsection{Wet Chemical Etching}

The exposed samples are etched in a $40 \%$ aqueous $\mathrm{KOH}$ solution at $60{ }^{\circ} \mathrm{C}$. This anisotropic etchant has an etch rate on the (100) Si crystal plane of about $0.25 \mu \mathrm{m} / \mathrm{min}$. Alternatively, for very small elements, a slower etch rate for precise time control is preferable and hence we use $\mathrm{KOH}$ at room temperature with an etch rate of only $\approx 1 \mu \mathrm{m} / \mathrm{h}$. Freestanding beams are achieved by appropriately orienting the FIB pattern at $45^{\circ}$ with respect to the (110) Si crystal plane.

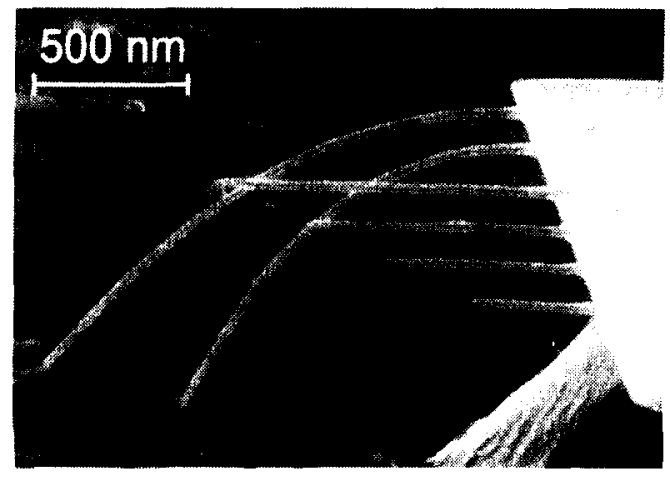

Figure 2. SEM of a series of $30 \mathrm{~nm}$ thin and 100 $\mathrm{nm}$ wide Si cantilevers with length ranging from $0.5-2 \mu \mathrm{m}$. Only the shorter beams are stable enough to withstand the surface tension during the rinsing process.

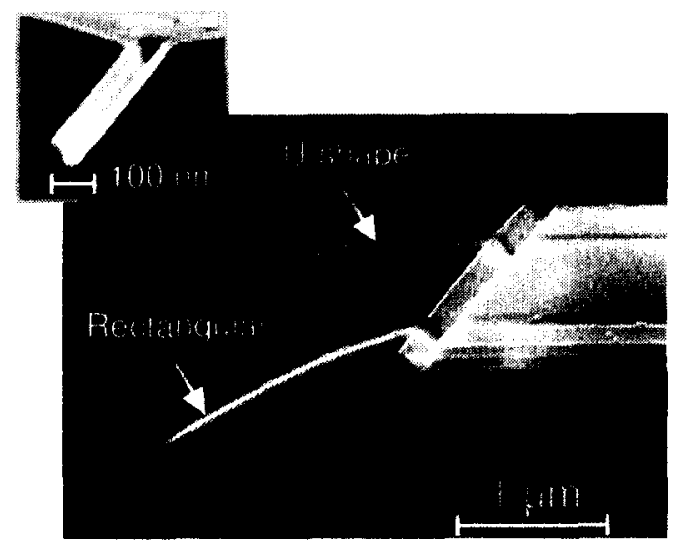

Figure 3. SEM of two $30 \mathrm{~nm}$ thin cantilevers with same length and mass, with rectangular and $U$ shaped cross section demonstrating the stabilizing effect by increasing the moment of inertia.

\section{NANOMECHANICAL ELEMENTS}

\subsection{Ultrahigh Frequency Cantilevers}

Using the technique described here, we have routinely fabricated freestanding $\mathrm{Si}$ beams with a thickness of $30 \mathrm{~nm}$ and a length and width ranging from $0.5-10 \mu \mathrm{m}$ and $0.1-1 \mu \mathrm{m}$, respectively. Fig. 2 shows a scanning electron micro- 
Table 1

Mechanical characteristics of two cantilever beams with a plane vs. a U-shaped cross section. Dimensions: $t=30 \mathrm{~nm}, b_{1}=100 \mathrm{~nm}, d=10 \mathrm{~nm}, b_{2}=b_{1}-2 d=80 \mathrm{~nm}, l=1 \mu \mathrm{m}$, as denoted in Fig. 1.

\begin{tabular}{lccc}
\hline & plane shape & U-shape & ratio $^{\mathrm{a}}$ \\
\hline mass $m[\mathrm{~kg}]$ & $6.9^{-18}$ & $6.9^{-18}$ & 1 \\
moment of inertia $I\left[\mathrm{~m}^{4}\right]$ & $2.25^{-31}$ & $2.7^{-29}$ & 120 \\
spring constant $k[\mathrm{~N} / \mathrm{m}]^{\mathrm{b}}$ & 0.11 & 13.7 & 120 \\
resonance frequency $f[\mathrm{MHz}]$ & 20 & 224 & 11 \\
\hline
\end{tabular}

${ }^{a}$ U-shape vs. normal shape ratio

${ }^{\mathrm{b}}$ calculated using the elastic module $E=1.7 \times 10^{11} \mathrm{~N} / \mathrm{m}^{2}$

graph (SEM) of a series of $100 \mathrm{~nm}$ wide cantilevers. The beams longer than $1.2 \mu$ could not withstand the surface tension forces during the wet rinsing process. The picture shows clearly how fragile the ultrathin elements are. For the nano-engineering of devices with a higher complexity, this weak point can be circumvented as described in the following.

Usually the FIB is used to dope a shallow surface layer. This defines the cross section of the underetched beam to be a rectangular shape (cf. left column of Fig. 1). However, using the FIB to mill a groove results in a U-shape cross section after the KOH with a much higher moment of inertia $I$ of the beam (cf. right column of Fig. 1) which plays an important role in the stability of the ultrathin cantilevers. Referring to the notation in Fig. 1, we can give the moment $I$ of a rectangular cross section as $I_{R}=\left(b_{1} t^{3}\right) / 12$, whereas for a U-shaped cross section due to the vertical sidewalls, this moment increases by several orders of magnitude, and can be expressed by [7]

$$
\begin{aligned}
I_{U}= & \frac{b_{2}}{3}(d+t)^{3}-\frac{d^{3}}{3}\left(b_{2}-2 t\right) \\
& -A(d+t-y)^{2},
\end{aligned}
$$

where $A=t b_{2}+2 t d$ and

$y=\frac{b_{2} t^{2}+2 t d(2 t+d)}{2\left(t b_{2}+2 t d\right)}$.

With increasing $I$, the spring constant $k$ of a cantilever of length $l$ and an elastic module $E$, given by $k=3 E I / l^{-3}$, is increased by the same amount. The resonance frequency is given by $f=(1 / 2 \pi) \sqrt{k / m}$. For example, a rectangular cantilever having a length, width, and thickness of $1 \mu \mathrm{m}, 100 \mathrm{~nm}$, and $30 \mathrm{~nm}$, respectively, results in a spring constant of $k_{\mathrm{R}}=0.11 \mathrm{~N} / \mathrm{m}$ and $f_{\mathrm{R}}=20 \mathrm{MHz}$, whereas a U-shaped cantilever with same length and mass yields $k_{\mathrm{U}}=13.7 \mathrm{~N} / \mathrm{m}$ and $f_{\mathrm{U}}=224 \mathrm{MHz}$, which is 120 and 11 times higher, respectively. Table 1 summarizes this result.

Figure 3 illustrates the mechanical stabilizing effect of a U-shaped cantilever cross section compared to a rectangular shape. It shows a SEM of two cantilevers with the same length and mass. The ultrathin cantilever with the rectangular cross section cannot withstand the surface attractive forces and is bent down. These forces probably originate from the surface tension during the rinsing process, which, in contrast, the 100-fold stiffer beam can withstand.

\subsection{Nanocups and Containers}

By extending the vertical FIB milling to several micrometers, real three-dimensional devices with vertical sidewalls of $30 \mathrm{~nm}$ thickness can be fabricated. Nanocups with ultrasmall volumes of $\approx 3 \times 10^{-8} n \ell$ have been fabricated by spot-mode milling a hole $200 \mathrm{~nm}$ in diameter and a few micrometers deep. Subsequent etching of the surrounding material leaves very stable elements that can also serve as anchor points for smaller elements such as cantilevers. Figure 4 shows a sequence of SEM images of this process at various stages.

\subsection{Nanomechanical Engineering of Com- plex Structures}

By combining the arbitrary pattern/milling of direct-write FIB exposure and anisotropic $\mathrm{KOH}$ etching, complex devices can be built. Figure 5 


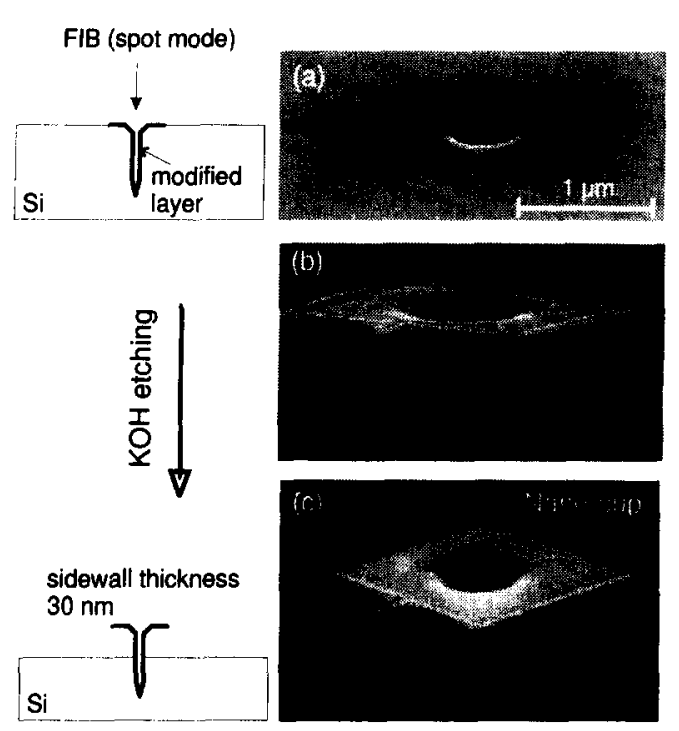

Figure 4. Process sequence for a nanocup (a) after FIB milling a hole in spot mode and shallow area exposure, (b) after $10 \mathrm{~min}$, and (c) after 15 min $\mathrm{KOH}$ etching.

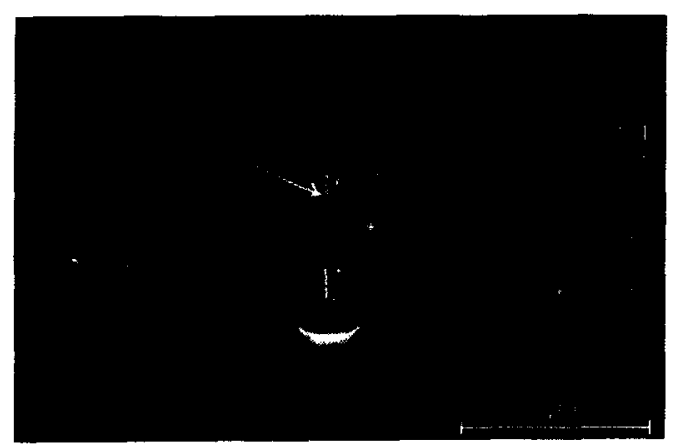

Figure 5. SEM of a ultrasmall torsional micromirror fabricated by 3D FIB milling, surface doping and $\mathrm{KOH}$ etching.

shows a SEM of a ultrasmall mirror element, where a $2 \times 2 \mu \mathrm{m}^{2}$ plate is suspended via two torsional hinges to a frame supported by four posts. This element could be used as a pixel in a deflection mirror matrix display with ultrahigh switch- ing speed. It shows nicely the feasibility of arbitrarily shaped three-dimensional devices by combined FIB/KOH fabrication.

\section{CONCLUSION}

In summary, we have shown novel micro/nanomechanical elements fabricated by a combination of FIB patterning/milling and $\mathrm{KOH}$ etching, such as cantilevers, cups, and more complex structures. We were able to improve the stability of the ultrathin elements by a factor of over 100 by using the FIB milling to shape a cross section with increased moment of inertia, which in turn results in mechanical resonance frequencies in the hundreds of $\mathrm{MHz}$ range.

Truly three-dimensional devices are feasible with the technique presented here. We believe that the presented approach has the potential to become a key process in the fabrication of mechanical systems in the nanometer range.

\section{Acknowledgement}

We are pleased to acknowledge P. Domeisen, $\mathrm{H}$. Rohrer and H. Rothuizen for stimulating discussions.

\section{REFERENCES}

1. I.L. Berry and A.L. Caviglia, J. Vac. Sci. Technol. B 1(4) (1983) 1059-1061.

2. A.J. Steckl, H.C. Mogul and S. Mogren, Appl. Phys. Lett. 60(15) (1992) 1833-1835.

3. Wei Chen et al., Mat. Res. Soc. Symp. Proc. 279 (1993).

4. A. Bohg, J. Electrochem. Soc. 118 (1971) 401

5. J.B. Bean, IEEE Trans. Electron Devices ED25 (1978) 1185.

6. FIB200 from FEI.

7. W.C. Young, Roark's formulas for stress and strain (McGraw-Hill, New York, 1989). 